\title{
Targeting TMEM16A to reverse vasoconstriction and remodelling in idiopathic pulmonary arterial hypertension
}

\author{
Rita Papp ${ }^{1,10}$, Chandran Nagaraj ${ }^{1,10}$, Diana Zabini, ${ }^{1,2}$, Bence M. Nagy ${ }^{1}$, \\ Miklós Lengyel ${ }^{3}$, Davor Skofic Maurer ${ }^{2}$, Neha Sharma ${ }^{1}$, \\ Bakytbek Egemnazarov ${ }^{1}$, Gabor Kovacs ${ }^{1,4}$, Grazyna Kwapiszewska ${ }^{1}$, \\ Leigh M. Marsh (1) ${ }^{1}$, Andelko Hrzenjak ${ }^{1,4}$, Gerald Höfler ${ }^{5}$, Miroslava Didiasova ${ }^{6}$, \\ Malgorzata Wygrecka ${ }^{6}$, Laura K. Sievers (10 ${ }^{7}$, Peter Szucs ${ }^{8}$, Péter Enyedi $^{3}$, \\ Bahil Ghanim ${ }^{9}$, Walter Klepetko ${ }^{9}$, Horst Olschewski ${ }^{4}$ and Andrea Olschewski ${ }^{1,2}$
}

@ERSpublications

TMEM16A plays a central role in the pathological mechanisms underlying the depolarisation, vasoconstriction and proliferation of PASMCs, contributing to the increased pulmonary vascular resistance in PAH, thus providing a novel target for PAH therapy http://ow.ly/3Rs330o3CUy

Cite this article as: Papp R, Nagaraj C, Zabini D, et al. Targeting TMEM16A to reverse vasoconstriction and remodelling in idiopathic pulmonary arterial hypertension. Eur Respir J 2019; 53: 1800965 [https://doi.org/10.1183/13993003.00965-2018].

ABSTRACT Our systematic analysis of anion channels and transporters in idiopathic pulmonary arterial hypertension (IPAH) showed marked upregulation of the $\mathrm{Cl}^{-}$channel TMEM16A gene. We hypothesised that TMEM16A overexpression might represent a novel vicious circle in the molecular pathways causing pulmonary arterial hypertension (PAH).

We investigated healthy donor lungs $(n=40)$ and recipient lungs with IPAH $(n=38)$ for the expression of anion channel and transporter genes in small pulmonary arteries and pulmonary artery smooth muscle cells (PASMCs).

In IPAH, TMEM16A was strongly upregulated and patch-clamp recordings confirmed an increased $\mathrm{Cl}^{-}$ current in PASMCs $(n=9-10)$. These cells were depolarised and could be repolarised by TMEM16A inhibitors or knock-down experiments $(n=6-10)$. Inhibition/knock-down of TMEM16A reduced the proliferation of IPAH-PASMCs $(n=6)$. Conversely, overexpression of TMEM16A in healthy donor PASMCs produced an IPAH-like phenotype. Chronic application of benzbromarone in two independent animal models significantly decreased right ventricular pressure and reversed remodelling of established pulmonary hypertension.

Our findings suggest that increased TMEM16A expression and activity comprise an important pathologic mechanism underlying the vasoconstriction and remodelling of pulmonary arteries in $\mathrm{PAH}$. Inhibition of TMEM16A represents a novel therapeutic approach to reverse remodelling in PAH.

This article has supplementary material available from erj.ersjournals.com

Received: May 242018 | Accepted after revision: Feb 212019

Copyright @ERS 2019 


\section{Introduction}

Idiopathic pulmonary arterial hypertension (IPAH) is a rare disease characterised clinically by the constriction of precapillary pulmonary arteries and associated with irreversible remodelling. The resulting increase in pulmonary arterial pressure leads to right ventricular hypertrophy and eventually death from right heart failure. Excess proliferation of pulmonary arterial endothelial cells and smooth muscle cells (SMCs) is one of the final, common pathological outcomes of distinct pathways involved in the development of IPAH. The pathophysiologic mechanism involves several signalling pathways [1, 2], including depolarisation and $\mathrm{Ca}^{2+}$ overload of the pulmonary artery SMCs (PASMCs) [3].

Both membrane depolarisation and $\mathrm{Ca}^{2+}$ overload are the result of the altered expression and function of different ion channels and transporters, as well as $\mathrm{Ca}^{2+}$ handling proteins. Decreased gene expression or loss-of-function mutation of voltage-gated $[4,5]$ and two-pore domain $\mathrm{K}^{+}$channels $[6,7]$, and increased expression of non-selective cation channels [8-10], the $\mathrm{Na}^{+} / \mathrm{Ca}^{2+}$ exchanger [11] and $\mathrm{Ca}^{2+}$ handling proteins [12] have been demonstrated in the PASMCs of IPAH patients. It has long been known that $\mathrm{Ca}^{2+}$-activated $\mathrm{Cl}^{-}$ currents are present in SMCs [13], but little attention has been paid to anion channels and transporters in IPAH. Recent reports that identify the encoding genes [14-16] as well as selective blockers of these channels [17, 18] have provided new tools to assess the role of anion currents in vascular function as well as in pathological states.

The $\mathrm{Ca}^{2+}$-activated $\mathrm{Cl}^{-}$channel TMEM16A, encoded by the gene ANO1, is active at the physiological resting membrane potential in human PASMCs $(\sim-50 \mathrm{mV})$. Because the intracellular $\mathrm{Cl}^{-}$concentration of a PASMC is relatively high ( 45 mM [19]), when TMEM16A channels open, a $\mathrm{Cl}^{-}$efflux depolarises the PASMC, with subsequent $\mathrm{Ca}^{2+}$ influx. TMEM16A is expressed in the pulmonary arteries of several species, including humans [20], but its function in human PASMCs and its involvement in pulmonary circulatory pathology are poorly understood.

We provide evidence that the expression and function of TMEM16A is significantly increased in IPAH patients, resulting in depolarisation and hyper-proliferation of PASMCs. Chronic administration of the TMEM16A inhibitor benzbromarone (BBR), approved for the treatment of gout in humans, reversed both increased pulmonary arterial pressure and vascular remodelling in animal models of pulmonary hypertension $(\mathrm{PH})$. We present a new approach focusing on the TMEM16 $\mathrm{A} \mathrm{Cl}^{-}$channel, which might be an important therapeutic target in severe $\mathrm{PH}$.

These studies have been reported in part in poster form at the International Conference of the American Thoracic Society in Washington, 2017, and the American Heart Association Scientific Sessions in Chicago, 2014.

\section{Methods}

Please refer to the supplementary material for full experimental details, including information on primary human cell isolation, in vitro experiments, electrophysiology studies, animal models of $\mathrm{PH}$ and assessment of their endpoints.

\section{Human lung samples}

Donor/IPAH patient characteristics are given in supplementary table E1. The clinical trial concerning the acute haemodynamic effects of BBR is registered at ClinicalTrials.gov under NCT02790450.

\section{Laser capture microdissection of pulmonary arteries}

Laser capture microdissection (LCM) of 17 donor lungs and 14 lungs from IPAH patients was performed as previously described [21]. Primer pairs (Eurofins, Graz, Austria) are summarised in supplementary table E2.

\section{Animal models of $\mathrm{PH}$}

All animal studies conformed to the EU guidelines 2010/63/EU and were approved by the University Animal Care Committee; the federal authorities for animal research approved the study protocol (approval numbers: BMWFW-66.010/0144-WF/V/3b/2014, BMWFW-66.010/0076-WF/V/3b/2015).

Affiliations: 'Ludwig Boltzmann Institute for Lung Vascular Research, Graz, Austria. ${ }^{2}$ Dept of Physiology, Medical University of Graz, Graz, Austria. ${ }^{3}$ Dept of Physiology, Faculty of Medicine, Semmelweis University, Budapest, Hungary. "Division of Pulmonology, Dept of Internal Medicine, Medical University of Graz, Graz, Austria. ${ }^{5}$ Dept of Pathology, Medical University of Graz, Graz, Austria. ${ }^{6}$ Dept of Biochemistry, Universities of Giessen and Marburg Lung Center, Giessen, Germany. ${ }^{7}$ Medical Clinic D, University Clinic of Münster, Münster, Germany. ${ }^{8}$ Dept of Anatomy, Histology and Embryology, University of Debrecen, Debrecen, Hungary. ${ }^{9}$ Division of Thoracic Surgery, Dept of Surgery, Medical University of Vienna, Vienna, Austria. ${ }^{10}$ Contributed equally.

Correspondence: Andrea Olschewski, Stiftingtalstrasse 24, Graz, A-8010, Austria.

E-mail: andrea.olschewskidlvr.lbg.ac.at 


\section{Statistical analyses}

Data are shown either as individual data plots with median, or summarised as mean \pm SEM. Statistical analyses were performed using Prism 6.0 (GraphPad Software, La Jolla, CA, USA). Statistical analyses were two-sided for all datasets, and p-values $<0.05$ were considered significant.

\section{Results}

\section{Upregulation of TMEM16A in the PASMCS of IPAH patients}

We evaluated the expression of anion channels and transporter genes in the laser capture microdissected pulmonary artery (LCM-PA) of freshly explanted healthy donors and of lung recipients with IPAH. The expression pattern of nine channels and five transporters is shown in figure 1a. The mRNA for the $\mathrm{Ca}^{2+}$-activated $\mathrm{Cl}^{-}$channel TMEM16A gene (ANO1) was upregulated in the LCM-PA (figure 1a, b) as well as in primary PASMCs isolated from IPAH patients (figure 1c). No significant regulation of the other channels/transporters was seen, except for the $\mathrm{Cl}^{-}$channel cystic fibrosis transmembrane conductance regulator (CFTR), which showed lower expression in the pulmonary artery of IPAH patients (figure 1a, supplementary figure E1). Immunofluorescent staining for $\alpha$-smooth muscle actin and TMEM16A on lung sections from healthy donors and IPAH patients (figure $1 \mathrm{~d}$, supplementary figure E2) showed the presence of TMEM16A in the medial layer of the pulmonary artery, both in donor lungs and in the remodelled arteries of IPAH lungs, as well as in primary PASMCs isolated from both donors and IPAH patients (supplementary figure E3). Western blots detected a marked increase of TMEM16A in the membrane protein fraction of PASMCs from IPAH patients (figure 1e-g). Accordingly, whole-cell voltage clamp measurements showed an increased $\mathrm{Ca}^{2+}$-activated $\mathrm{Cl}^{-}$current (IClCa) in primary PASMCs from IPAH patients compared to the PASMCs from healthy donors (figure $1 \mathrm{~h}$ ).

Next, we addressed upstream events that are likely to regulate TMEM16A. Because the recently reported zinc metalloprotease calcium-activated chloride channel activator 1 (CLCA1) was shown to activate chloride currents in a paracrine fashion and to stabilise cell surface TMEM16A in HEK293T cells exogenously $[22,23]$, we investigated CLCA1 protein levels in the plasma and lungs of IPAH patients. We found that CLCA1 was not significantly altered in plasma or lung homogenate of IPAH patients compared to donors (supplementary figure E4A, B). Demographic and haemodynamic data of the patients whose blood plasma or lung homogenate was used for the CLCA1 concentration measurements are shown in supplementary tables E3 and E1, respectively. Treating the PASMCs of healthy donors with CLCA1-containing conditioned medium did not affect the resting membrane potential (supplementary figure E4C, D). We further quantified the expression of three exons reported to be subject to alternative splicing, because these may influence the biophysical properties of TMEM16A channels. We found no difference in the expression of splice variants between the PASMCs of donors and IPAH patients (supplementary figure E5). Accordingly, there was no apparent difference in the biophysical properties of IClCa in the voltage clamp recordings (figure 1f, supplementary figure E6). Finally, in silico analysis predicted hypoxia-inducible factor $1 \alpha(\mathrm{HIF}-1 \alpha)$ binding sites in the promoter region of the TMEM16A gene. Hypoxia for $48 \mathrm{~h}$ increased the amount of TMEM16A protein in the primary PASMCs (supplementary figure E7A, B) and formed functional channels in the cell membranes, as demonstrated by an increased whole-cell current (IClCa) in the cells exposed to hypoxia (supplementary figure E7C). Thus, in PASMCs obtained from healthy donors, chronic hypoxia induced the features of IPAH, including enhanced expression of TMEM16A protein and an increased whole-cell $\mathrm{Cl}^{-}$current.

\section{Upregulated TMEM16A causes chronic PASMC membrane depolarisation in IPAH}

In order to determine the role of TMEM16A in the membrane potential in human PASMCs, we controlled the expression of TMEM16A and subsequently examined its impact in human PASMCs. When TMEM16A was silenced, TMEM16A mRNA, total protein and IClCa in primary PASMCs decreased compared to in PASMCs treated with non-silencing control RNA from donors and IPAH patients (figure $2 \mathrm{a}-\mathrm{c}$, supplementary figure $\mathrm{E} 8$, representative current traces shown in supplementary figure E6). Similarly, BBR, a recently identified inhibitor of TMEM16A channels, significantly decreased whole-cell IClCa measured in primary PASMCs of both donors and IPAH patients (figure $2 \mathrm{~d}$, supplementary figure E6). This BBR-sensitive current was abolished by silencing of TMEM16A (supplementary figure E9). The resting membrane potential $(\mathrm{Em})$ of primary PASMCs isolated from IPAH patients was significantly more depolarised than the Em of donor PASMCs (figure 2e, f). BBR reversed the Em of IPAH-PASMCs to the levels of PASMCs isolated from healthy donors, whereas it had no effect on the PASMCs of donors (figure 2e). Another structurally non-related TMEM16A blocker, T16Ainh-A01, did not significantly change Em (figure 2e). Silencing of TMEM16A in IPAH-PASMCs rescued (repolarised) the Em of IPAH-PASMCs without significantly affecting donor PASMCs (figure 2f). As a second approach, we overexpressed TMEM16A in human donor PASMCs. The overexpression of TMEM16A was verified by an increase in TMEM16A mRNA $48 \mathrm{~h}$ post-transfection (figure $2 \mathrm{~g}$ ), and by an increased TMEM16A total protein signal (figure $2 \mathrm{~h}$ ) accompanied by an elevated IClCa (figure 2i). Representative current traces are shown in 

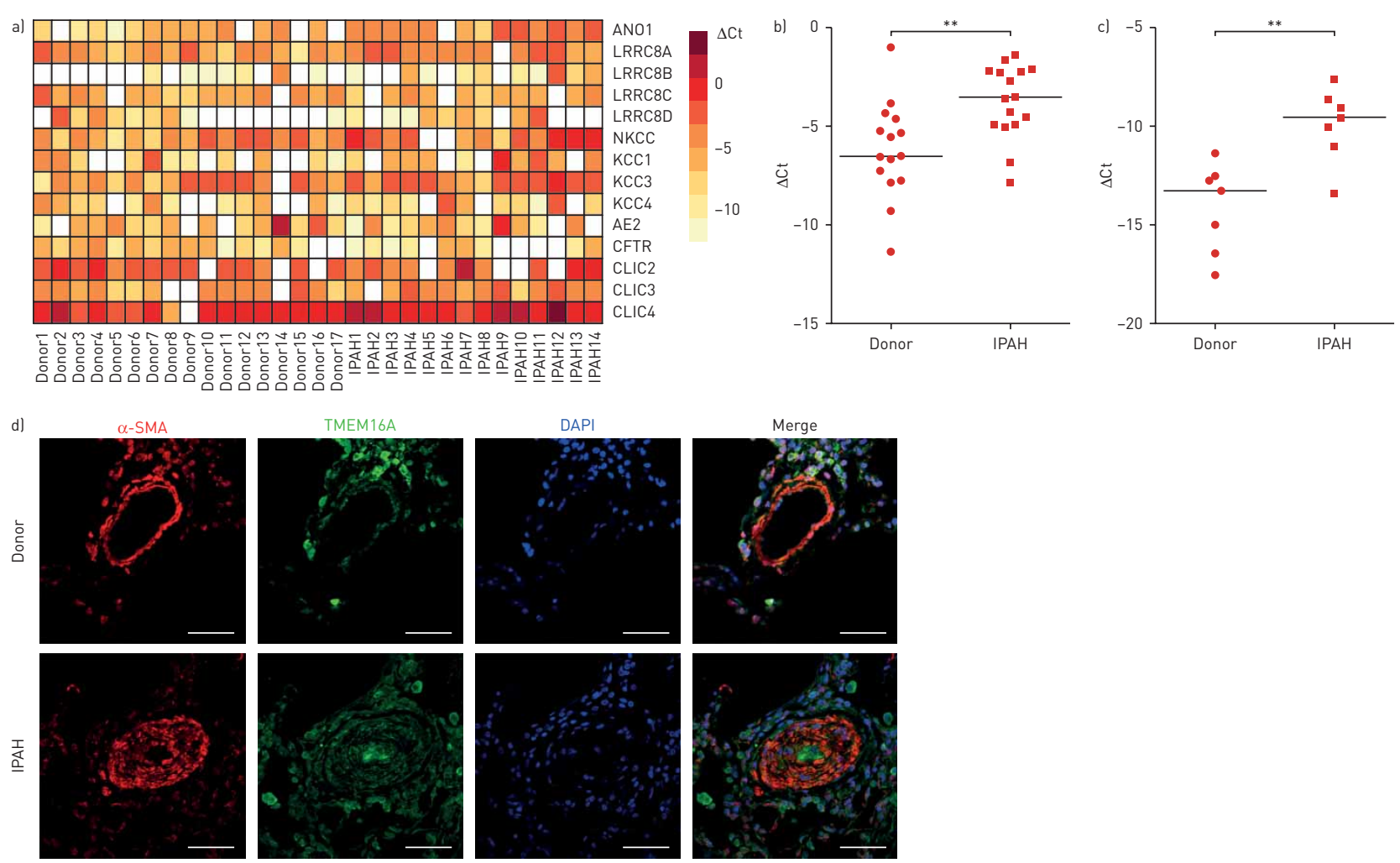

e)
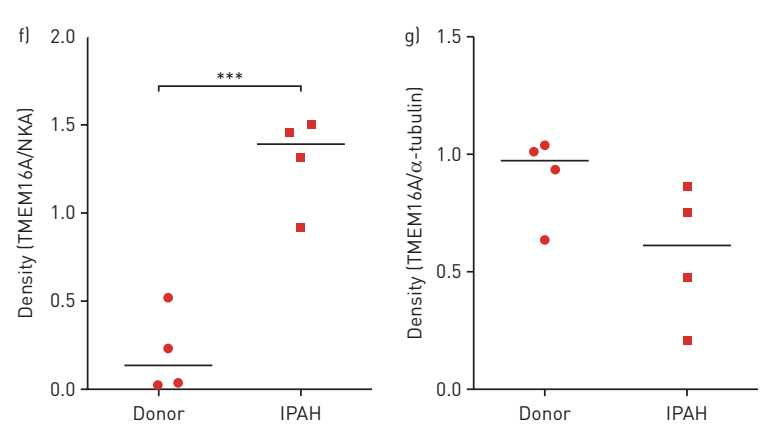

h)

Donor

IPAH

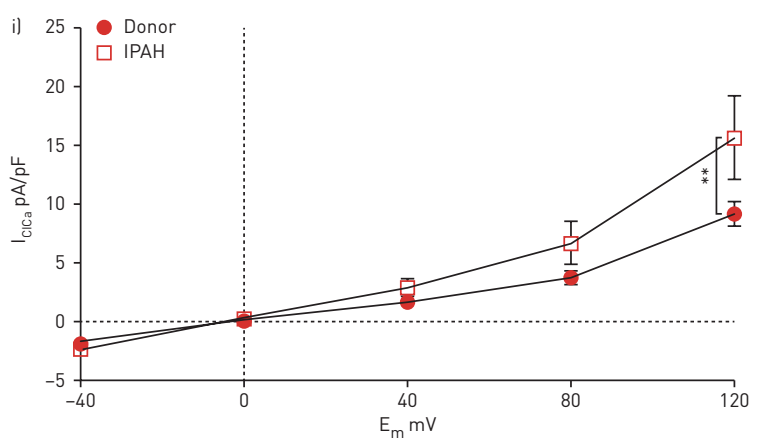

FIGURE 1 Upregulation of TMEM16A and increased $\mathrm{Ca}^{2+}$-activated $\mathrm{Cl}^{-}$current (ICICa) in the pulmonary artery smooth muscle cells (PASMCs) of idiopathic pulmonary arterial hypertension (IPAH) patients. a) Quantitative reverse transcriptase PCR heat map depicting the expression of nine $\mathrm{Cl}^{-}$channel and five $\mathrm{Cl}^{-}$transporter genes in laser capture microdissected pulmonary arteries (LCM-PA) from 17 healthy donors and 14 IPAH patients. $\triangle \mathrm{Ct}$ values, calculated by normalising the expression of target genes to $\beta 2$ microglobulin expression, are shown. White boxes indicate that no PCR product was detected. b, c) Expression of the TMEM16A mRNA in LCM-PA (b) and in primary PASMCs (c) isolated from donors $(n=7-15)$ and IPAH patients $(n=7-16)$. $\Delta$ Ct values have been calculated as the difference between TMEM16A and $\beta 2$ microglobulin expression. d) Immunofluorescence staining for $\alpha$-smooth muscle actin $(\alpha-S M A)$ and TMEM16A in lung sections of donors and IPAH patients. Scale bar=50 $\mu$ m. e-g) Western blot comparing cell membrane expression of TMEM16A in the PASMCs of donors ( $n=4)$ and IPAH patients (n=4). Membrane and cytosolic protein fractions were separated by cell surface protein biotinylation. The $\mathrm{Na}^{+}-\mathrm{K}^{+}$ATPase $\alpha 1$ subunit (NKA $\left.\alpha 1\right)$ and $\alpha$-tubulin served as loading controls for membrane and cytosolic fractions, respectively. h, i) Representative whole-cell Icıca traces (h) and normalised current-voltage relationships measured with voltage clamp (i) in the PASMCs of healthy donors $(n=10)$ and IPAH patients $\left(n=9\right.$ for IPAH). ${ }^{* *}: p<0.01 ;{ }^{* * *}: p<0.001$; unpaired t-test used in b, c, f and g; two-way-ANOVA with Bonferroni post hoc test used in i. Parts c-i were performed with 4-7 different donors or IPAH patients. 

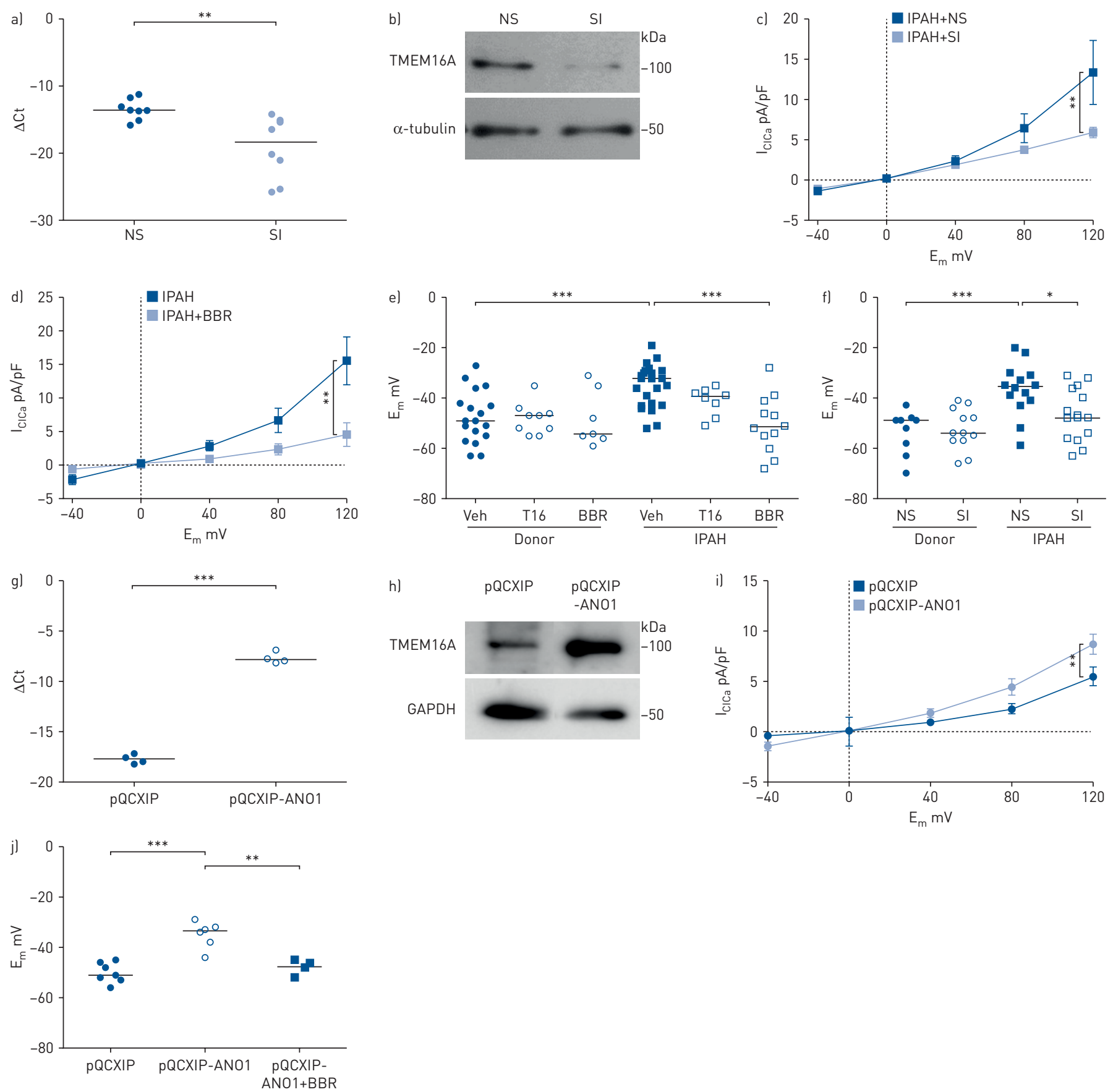

FIGURE 2 TMEM16A influences membrane potential in human pulmonary artery smooth muscle cells (PASMCs). a, b) TMEM16A mRNA expression (a) and total protein level (b) in PASMCs treated with either non-silencing control RNA (NS) or TMEM16A siRNA (SI). mRNA expression was studied $48 \mathrm{~h}$ post-transfection and is given as $\Delta \mathrm{Ct}$, calculated as the difference between TMEM16A and 32 microglobulin expression. c) Ca ${ }^{2+}$-activated $\mathrm{Cl}^{-} \mathrm{current}$ (ICICa) density in the PASMCs of idiopathic pulmonary arterial hypertension (IPAH) patients $72 \mathrm{~h}$ after treatment with non-silencing control RNA (NS, $\mathrm{n}=9$ ) or TMEM16A siRNA (SI, n=7). d) Effect of benzbromarone (BBR, $30 \mu \mathrm{M}$ ) on ICICa density in the PASMCs of IPAH patients (IPAH $n=9$, IPAH+BBR $n=7$ ). e) Membrane potential (Em) values obtained from PASMCs of healthy donors ( $n=7-18$ ) and IPAH patients ( $n=8-23$ ) in the absence (vehicle (Veh)) or presence of the TMEM16A blockers T16A $\mathrm{A}_{\text {inh-A01 }}(\mathrm{T} 16,10 \mu \mathrm{M})$ or BBR $(30 \mu \mathrm{M})$. f) Em of PASMCs $72 \mathrm{~h}$ after transfection with either non-silencing control RNA (NS) or TMEM16A siRNA (SI). g, h) TMEM16A mRNA (g) and protein (h) expression in donor PASMCs ( $\mathrm{n}=4$ ) after transfection with TMEM16A gene (AN01)-containing or empty pQCXIP plasmid (labelled pQCXIP-ANO1 and pQCXIP, respectively). i) IcICa current-voltage curves measured in the PASMCs of donors $72 \mathrm{~h}$ after transfection with empty $(n=6)$ or AN01-containing $(n=8) p Q C X I P$ plasmid. $j)$ Effect of transfection with pQCXIP-AN01 or pQCXIP plasmid on the Em of PASMCs isolated from donors (n=6-7). ${ }^{*}: p<0.05 ;{ }^{* *}: p<0.01 ;{ }^{* * *}$ : $p<0.001$; unpaired t-test used in a and g; two-way ANOVA with Bonferroni post hoc test in $c, d$ and i; one-way ANOVA with Bonferroni's multiple comparison test in e, $f$ and $j$. All experiments in figure 2 were performed with 4-8 different donors or IPAH patients. 
supplementary figure E6. TMEM16A overexpression brought about a significant depolarisation of PASMCs, which was reversed by applying BBR (figure $2 \mathrm{j}$ ). Note that both the whole-cell IClCa density and the Em recorded from the TMEM16A-overexpressing donor PASMCs mimicked the changes observed in PASMCs of IPAH patients.

\section{Acute vasorelaxant effect of the TMEM16A inhibitor BBR}

The finding that TMEM16A inhibition reverses membrane depolarisation in the PASMCs of IPAH patients prompted us to evaluate the effect of TMEM16A inhibition on the pulmonary circulation in both animal models and humans. To determine the effective dose for acute vasorelaxation, we examined the TMEM16A inhibitor-mediated pulmonary artery vasodilator response ex vivo. BBR caused a dose-dependent vasorelaxation of U-46619 pre-constricted isolated mouse and rat pulmonary artery (figure $3 \mathrm{a}, \mathrm{d}$ ). In the second approach we applied BBR in vivo. Under continuous in vivo haemodynamic monitoring, BBR was applied as an intravenous bolus in two different animal models of $\mathrm{PH}$ : in hypoxia-exposed mice (figure $3 \mathrm{~b}, \mathrm{c}$ ) and in monocrotaline (MCT)-treated rats (figure $3 \mathrm{e}, \mathrm{f}$ ). $\mathrm{BBR}$, at a concentration that effectively dilated pulmonary arteries ex vivo, caused a significant decrease in the right ventricular systolic pressure (RVSP) in both models without affecting RVSP in the control animals. TMEM16A was not regulated in the heart of $\mathrm{PH}$ animal models (supplementary figure E10). To assess the acute pulmonary vasodilatative potency of BBR in humans, we enrolled 10 patients with severe IPAH and administered $200 \mathrm{mg}$ BBR orally to eight of them as a single dose during a routine right heart catheter study. A dose of $200 \mathrm{mg}$ is the maximum approved single oral dose for the preventive treatment of gout in humans. Two patients were excluded, one owing to elevated pulmonary arterial wedge pressure $(>15 \mathrm{mmHg})$ and another because of elevated serum bilirubin levels $\left(>1.6 \mathrm{mg} \cdot \mathrm{dL}^{-1}\right)$. Demographic and haemodynamic data of the patients receiving BBR are described in supplementary table E4. There were no further changes in the pulmonary or systemic haemodynamic data (supplementary table E5). No clinical adverse effects were documented during the study.
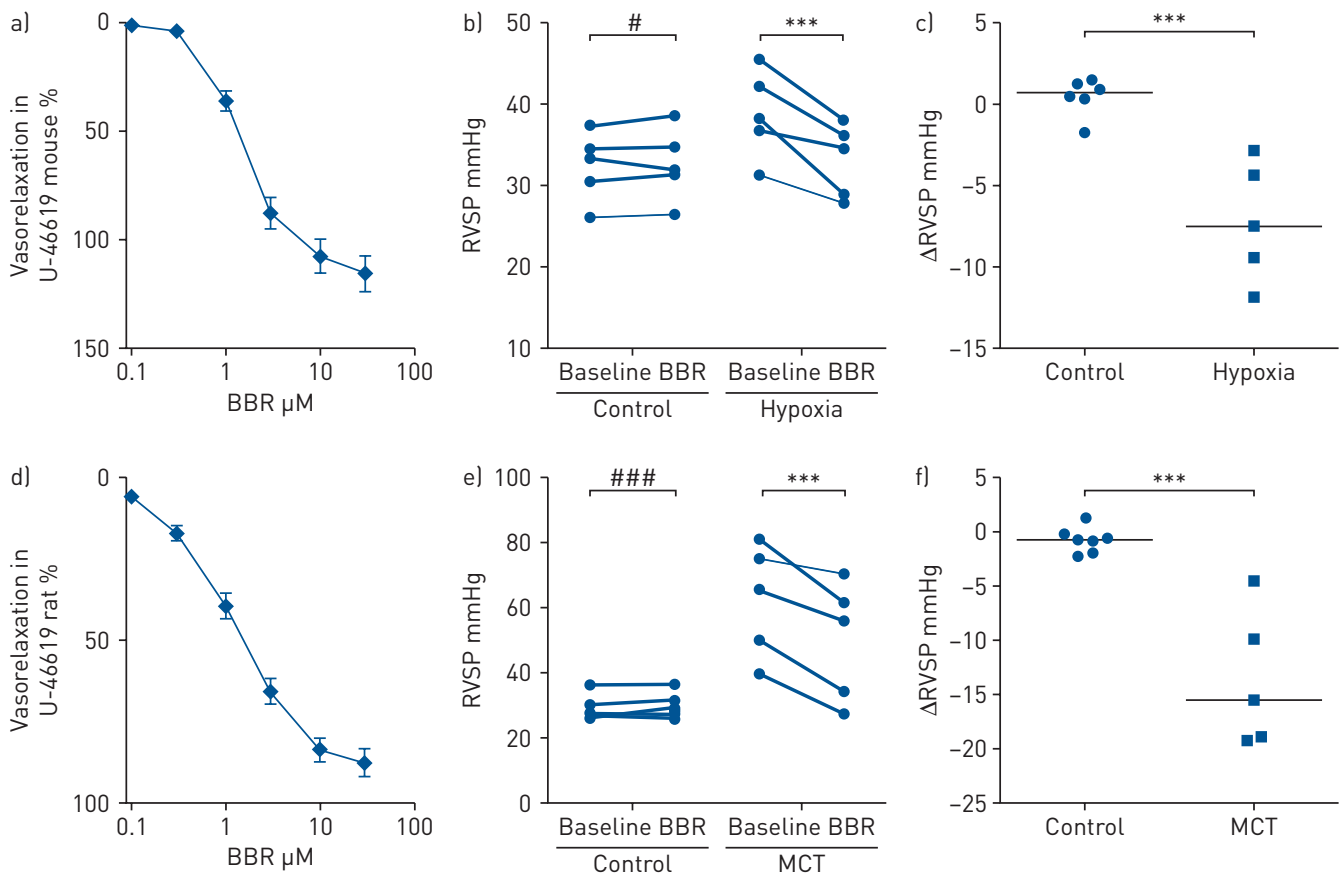

FIGURE 3 Acute TMEM16A inhibition relaxes the pulmonary artery both ex vivo and in vivo. Effect of the TMEM16A blocker benzbromarone (BBR) applied in cumulative doses on U-46619 (30 nM) pre-constricted mouse and rat pulmonary artery rings. a, d) Dose-response curves from mouse $(n=7)(a)$ and rat $(n=4)$ (d) pulmonary artery rings. b, c) Pre- and post-drug values (b) and maximal changes (c) in right ventricular systolic pressure (RVSP) measured with in vivo haemodynamic analysis during a single intravenous administration of $300 \mu \mathrm{M}$ BBR in mice exposed to 4 weeks of hypoxia or normoxia. e, f) Pre- and post-drug values (e) and maximal changes ( $\mathrm{f}$ ) in RVSP measured by means of in vivo haemodynamic analysis during a single intravenous administration of $300 \mu \mathrm{M}$ BBR in rats treated with monocrotaline (MCT) or vehicle. ${ }^{* * *}$ : $p<0.001$;

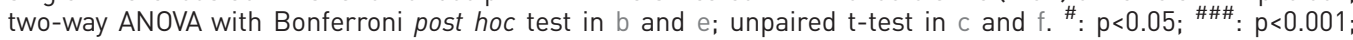
unpaired t-test in $b$ and $e$. 


\section{Chronic BBR treatment significantly decreases the RVSP and pulmonary arterial muscularisation} in the hypoxic mouse and MCT rat

To assess the therapeutic potency of BBR for reverse remodelling, we applied BBR and vehicle (Veh) as subcutaneous slow-release pellets in two different animal models of $\mathrm{PH}$. This dosage corresponds to previous studies in models of hyperuricaemia in rodents and monkeys [24-26]. A schematic diagram of the experiments using hypoxia-exposed mice is given in figure $4 \mathrm{a}$ and in the supplementary methods. RVSP was significantly decreased under long-term BBR treatment, without altering the systemic arterial pressure (figure $4 \mathrm{~b}, \mathrm{c}$ ). BBR led to a significant reduction of the hypoxia-induced pulmonary artery muscularisation (figure $4 \mathrm{~d}, \mathrm{e}$ ).

As in the mouse study, rats were randomised into a control group and two MCT-treated groups (figure 5a). MCT treatment caused a significant increase in right ventricular free wall thickness and

a)

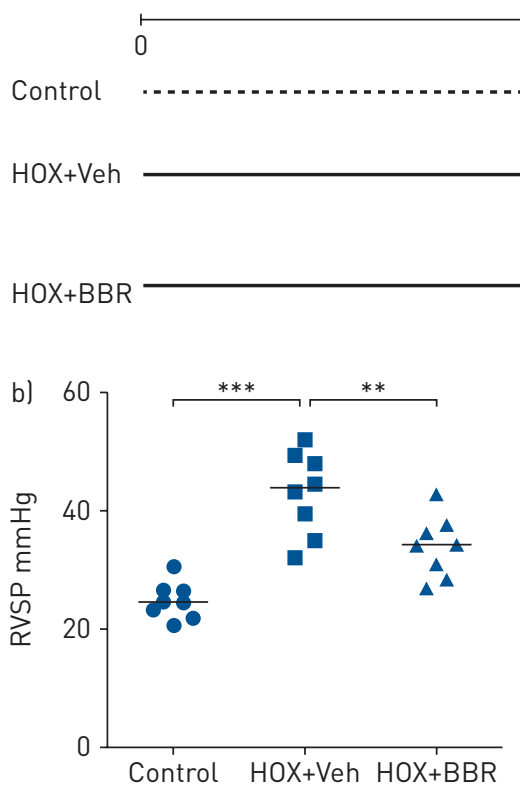

e)

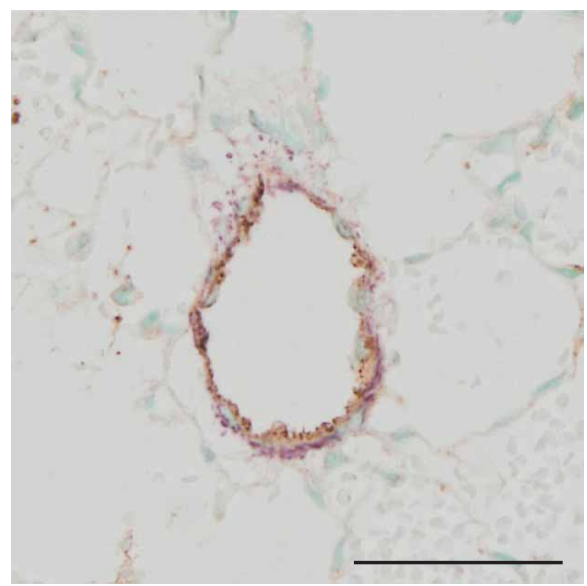

Time weeks

2

End of experiment (E):

Haemodynamics Acute BBR Organ collection
Veh

$$
\text { Hypoxia }\left(10 \% 0_{2}\right)
$$

Hypoxia $\left(10 \% \mathrm{O}_{2}\right)$
BBR

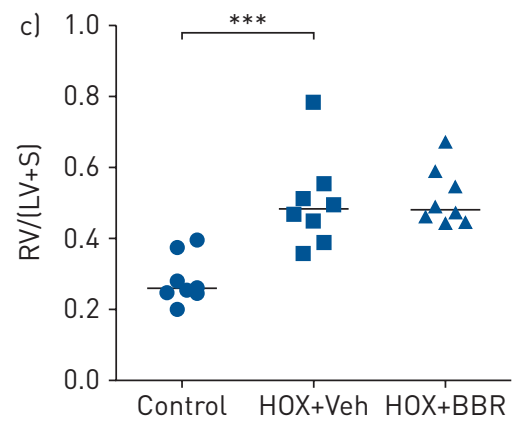

HOX+Veh

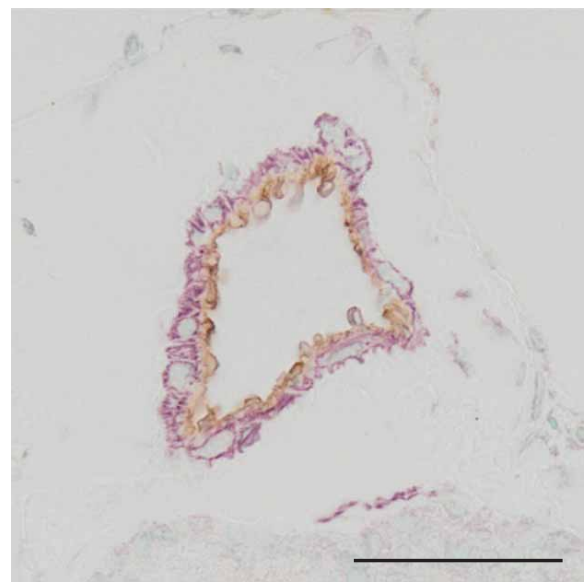

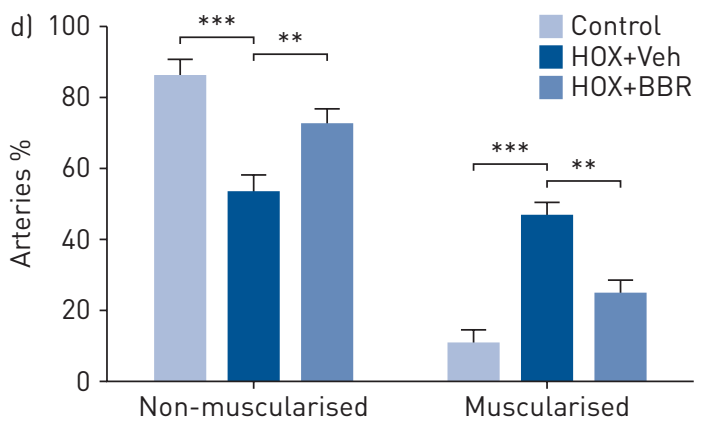

HOX+BBR

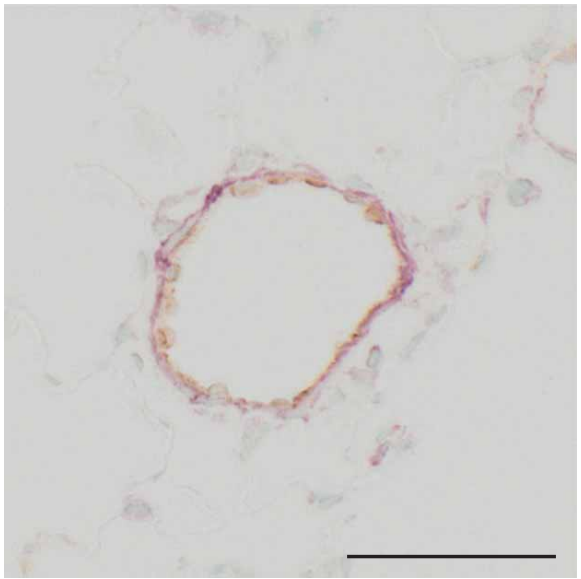

FIGURE 4 Chronic treatment with the TMEM16A inhibitor benzbromarone (BBR) reverses vasoconstrictive pulmonary artery remodelling in mice exposed to hypoxia. a) Schematic diagram of the experimental protocol. Mice were randomised into three groups. Groups HOX+Veh and HOX+BBR were exposed to 4 weeks of hypoxia, while mice in the control group $(n=8)$ were kept under normoxic conditions. After week 2 , subcutaneous slow-release pellets containing either vehicle $(\mathrm{HOX}+\mathrm{Veh}$ group, $n=8)$ or $B B R(\mathrm{HOX}+\mathrm{BBR}, \mathrm{n}=8)$ were implanted. At week 4 , all rats were subjected to haemodynamic analyses and organ collection, as indicated. b) Assessment of right ventricular systolic pressure (RVSP) with in vivo haemodynamic analysis and c) estimation of right ventricular hypertrophy (Fulton index; the weight ratio of the right ventricle (RV) to the left ventricle (LV) plus septum (S)). d) Analysis of pulmonary arterial remodelling expressed as the percentage change in the number of muscularised and non-muscularised arteries (Control $n=3, H O X+V e h n=5, H O X+B B R n=5$ ). e) Representative images showing the degree of muscularisation of resistance arteries. Scale bar $=50 \mu \mathrm{m} .{ }^{* *}: \mathrm{p}<0.01 ;{ }^{* * *}$ : $p<0.001$; one-way ANOVA with Bonferroni's multiple comparison test was used in b-d. 
a)

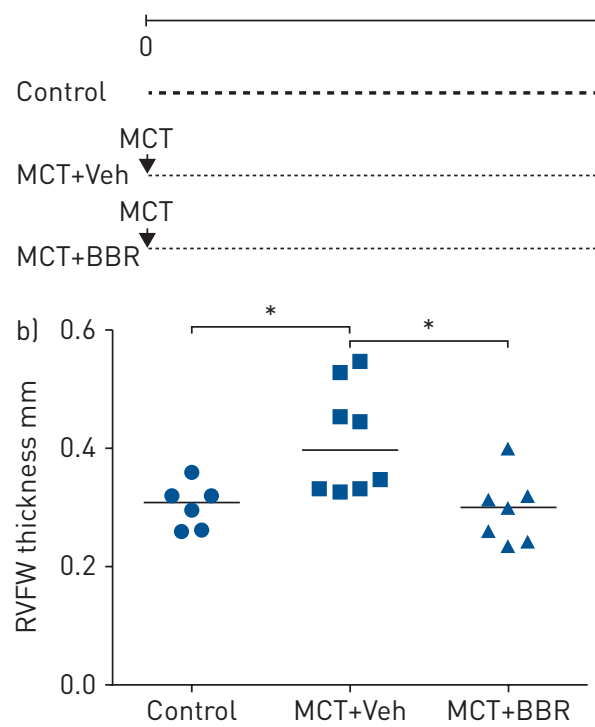

Time weeks

$$
2
$$

End of experiment $(E)$ :

Haemodynamics Acute BBR Organ collection

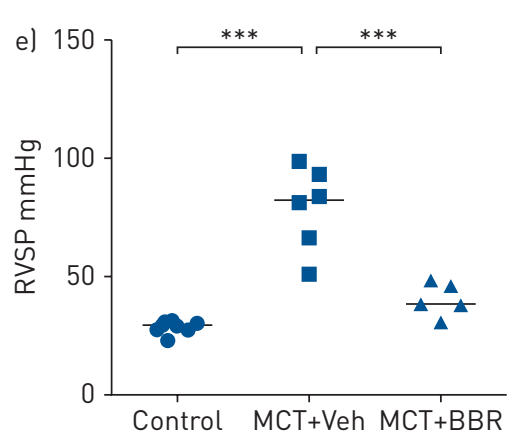

h)

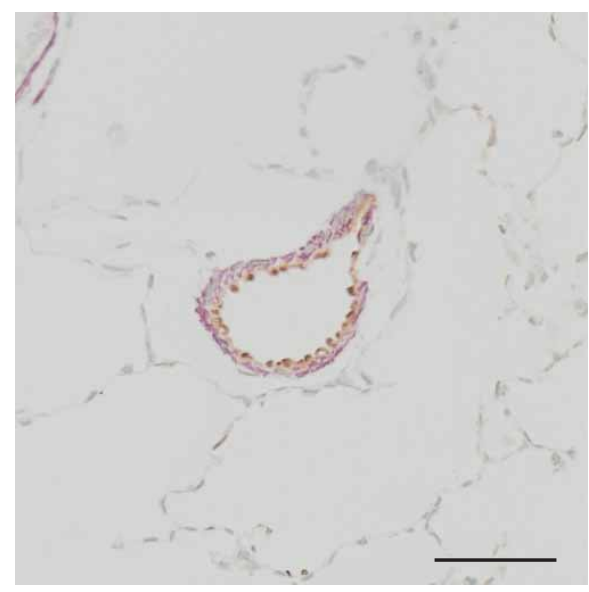

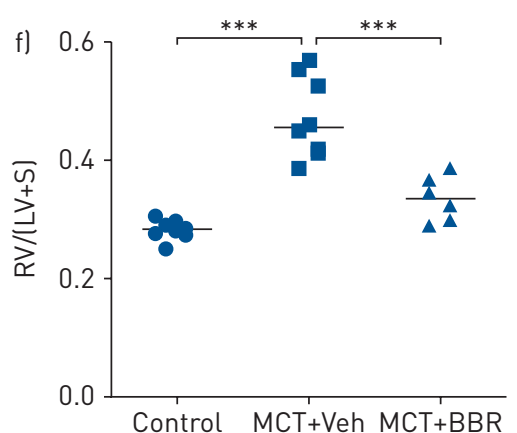

$\mathrm{MCT}+\mathrm{Veh}$

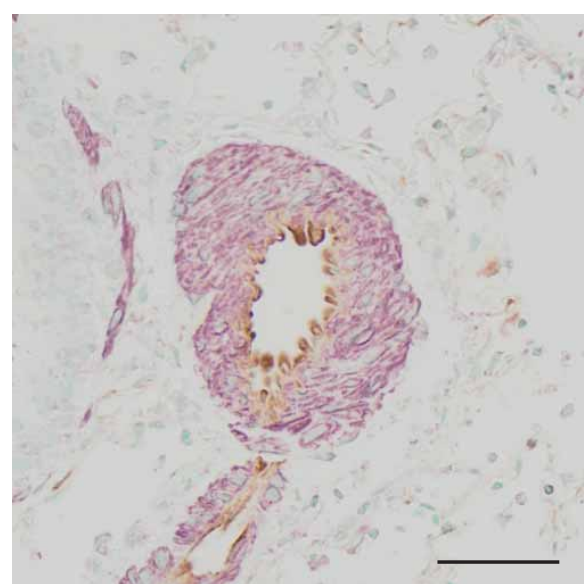

d)

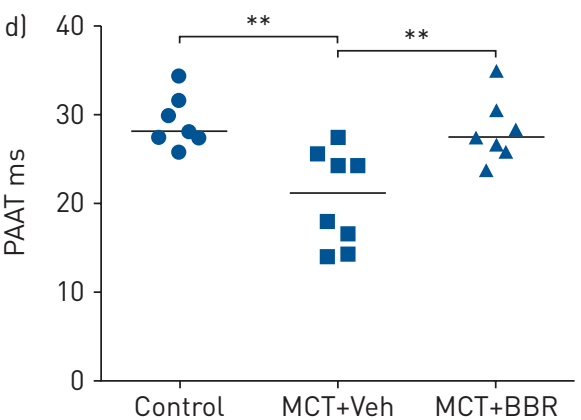

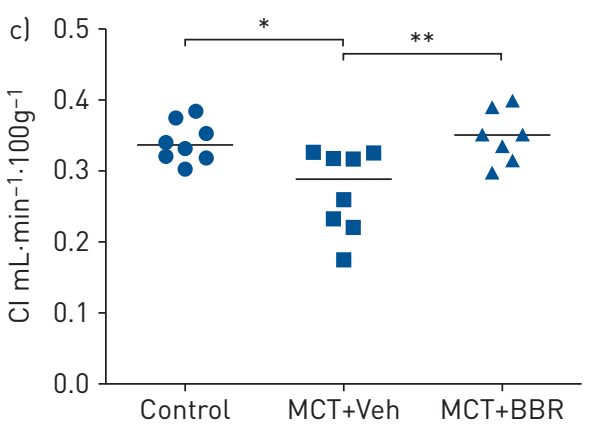

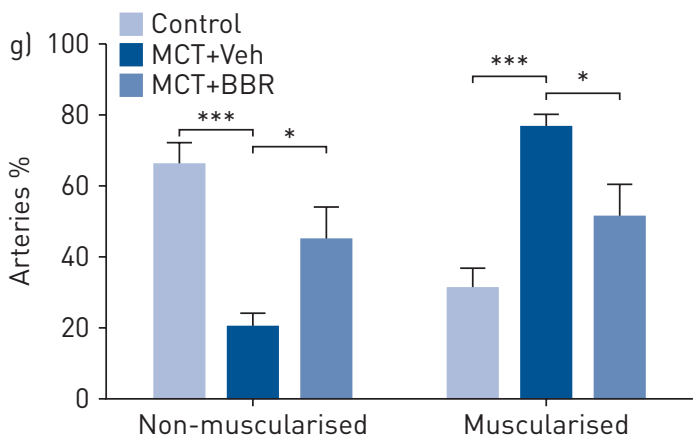

$\mathrm{MCT}+\mathrm{BBR}$

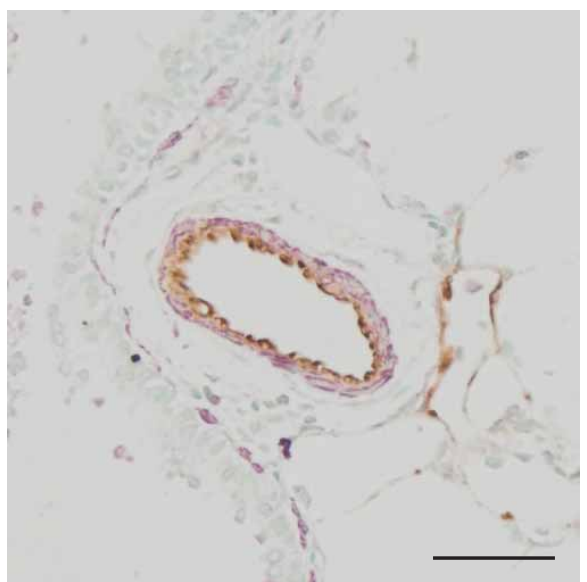

FIGURE 5 Chronic treatment with the TMEM16A inhibitor benzbromarone (BBR) reverses vasoconstrictive pulmonary artery remodelling in monocrotaline (MCT)-treated rats. a) Schematics of the experimental protocol. Rats were randomised into three groups. Groups MCT+Veh and $M C T+B B R$ ( $n=8$ each) were treated with MCT, while rats in the control group ( $n=8$ ) received vehicle. Two weeks after MCT treatment, subcutaneous slow-release pellets containing either vehicle (MCT+Veh group) or BBR (MCT+BBR) were implanted. At week 4, all rats were subjected to haemodynamic analyses and organ collection, as indicated. b-d) Echocardiographic assessment of the right ventricular free wall (RVFW) thickness (b), cardiac index (CI) (c) and pulmonary artery acceleration time (PAAT) (d) at week 4, 1 day before termination of the experiment. e) Right ventricular systolic pressure (RVSP) measured by in vivo haemodynamic analysis and f) calculation of right ventricular hypertrophy (Fulton index; the weight ratio of the right ventricle (RV) to the left ventricle (LV) plus septum (S)). g) Analysis of pulmonary arterial remodelling expressed as percentage change in the number of muscularised and non-muscularised arteries (Control $n=4, M C T+V e h n=7, M C T+B B R \quad n=5$ ). h) Representative images showing the degree of muscularisation of resistance arteries. Scale bar $=50 \mu \mathrm{m} .{ }^{*}: p<0.05 ;{ }^{* *}: p<0.01 ;{ }^{* * *}<<0.001$; one-way ANOVA with Bonferroni's multiple comparison test in b-g. 
decreases in pulmonary artery acceleration time and cardiac index as compared to the control group; however, after BBR treatment, MCT had nearly no effect, although BBR was only started after 2 weeks (nonsignificant versus control, figure $5 \mathrm{~b}-\mathrm{d}$ ). Further echocardiographic parameters are reported in supplementary table E6. Compared to the MCT+Veh group, RVSP and right ventricular hypertrophy were significantly decreased in the BBR-treated rats (figure $5 \mathrm{e}, \mathrm{f}$ ) without changes in systemic arterial pressure. The markedly reduced number of fully muscularised arteries and the increased number of non-muscularised arteries indicated that BBR induced a potent attenuation of vascular remodelling(figure $5 \mathrm{~g}, \mathrm{~h}$ ).

\section{Upregulated TMEM16A triggers PASMC proliferation}

Immunohistological staining of mouse and rat lung sections for the proliferation marker proliferating cell nuclear antigen (PCNA) showed an increased number of PCNA-positive nuclei in the medial layer of the pulmonary artery in both hypoxic mice (figure 6a) and MCT-treated rats (figure 6b), compared to the normoxic/Veh-treated controls. In parallel with the haemodynamic improvement, there were fewer PCNA-positive nuclei in the BBR-treated animals (figure 6a, b). In human donor and IPAH-PASMCs, both treatment with $\mathrm{BBR}$ and silencing RNA (siRNA) against TMEM16A led to a decrease in platelet-derived growth factor (PDGF)-BB-induced proliferation (figure $6 \mathrm{c}-\mathrm{f}$ ). We next investigated the direct effect of TMEM16A upregulation on the phosphorylation of cjun and cfos (figure 6). TMEM16A overexpression (figure 6j) enhanced cfos phosphorylation, followed by increased PASMC proliferation (figure $6 \mathrm{~g}-\mathrm{k}$ ), which is in line with our previous observation showing increased total cfos in the remodelled IPAH pulmonary arteries [27]. This suggests that TMEM16A-induced proliferation of human PASMCs is mediated by cfos phosphorylation and can be inhibited by BBR in both donor and IPAH-PASMCs (figure 6l-o).

\section{Discussion}

We found increased expression and activation of the $\mathrm{Ca}^{2+}$-activated $\mathrm{Cl}^{-}$channel TMEM16A in the PASMCs of IPAH patients that strongly contribute to the pathologic phenotype of these cells, as expressed by depolarisation, vasoconstriction and hyper-proliferation. Chronic treatment with the TMEM16A inhibitor BBR caused vasodilatation and strong attenuation of remodelling in two independent animal models of PH. Our work also shows that blocking or silencing of TMEM16A reversed the pathological membrane depolarisation in vitro in the PASMCs of IPAH patients, causing vasodilatation, and inhibition of PASMC proliferation.

Our systematic investigation of the compartment-specific regulation of $\mathrm{Cl}^{-}$channels and transporters in the pulmonary artery and in primary cultured PASMCs from IPAH patients showed strongly increased TMEM16A expression. This is in line with other reports [28-30] of upregulation of TMEM16A in the PASMCs of animal models of PH. Our study, however, is the first to demonstrate that these changes are very consistent in human PASMCs obtained from a large number of IPAH patients. Our study is also the first experimental investigation of the effects of TMEM16A inhibition and overexpression. PASMCs isolated from IPAH patients maintained their pathologic phenotype as they were depolarised and showed a TMEM16A upregulation similar to that found in the LCM-PA. This suggests that the upregulation of TMEM16A is among the pathologic mechanisms of IPAH. This concept is also supported by a previous study showing that endothelin-1 (ET-1), which plays an important role in PAH aetiology, upregulates the TMEM16A protein in human PASMCs [31]. Moreover, our in silico analysis predicted binding sites for the transcription factor HIF- $1 \alpha$ in the promoter region of TMEM16A, and a recent study on mouse coronary endothelial cells suggested that hypoxia may increase TMEM16A expression [32]. Accordingly, exposure to hypoxia increased sarcolemmal TMEM16A protein levels in the PASMCs of healthy donors above those of PASMCs kept under normoxic conditions, with functional consequences due to the generation of a greater $\mathrm{Ca}^{2+}$-activated $\mathrm{Cl}^{-}$current. Alternative splicing of the TMEM16A mRNA is another way to regulate the biophysical properties of TMEM16A channels: the presence of exons 6b, 13 and 15 is reported to influence $\mathrm{Ca}^{2+}$ and Em sensitivity as well as the speed of channel activation/ deactivation [33]. In contrast to the study by FORREST et al. [29] on the PASMCs of MCT-treated rats, our investigations did not show a differential expression of splice variants between the PASMCs of donors and IPAH patients; accordingly, there was no apparent difference in the biophysical properties of the recorded IClCa. Thus, although there are several potential mechanisms that could upregulate TMEM16A, a limitation of our study is that it cannot clearly define the signalling pathway leading to TMEM16 overexpression.

The functional consequences of TMEM16A overexpression are summarised in figure 7. Our electrophysiological studies confirmed previous reports that the PASMCs of IPAH patients are significantly depolarised compared to those of healthy donors [4]. Moreover, we demonstrated that pharmacological inhibition or silencing of TMEM16A reversed the membrane potential of IPAH-PASMCs back to the membrane potential range of healthy donors, whereas overexpression of TMEM16A depolarised the 

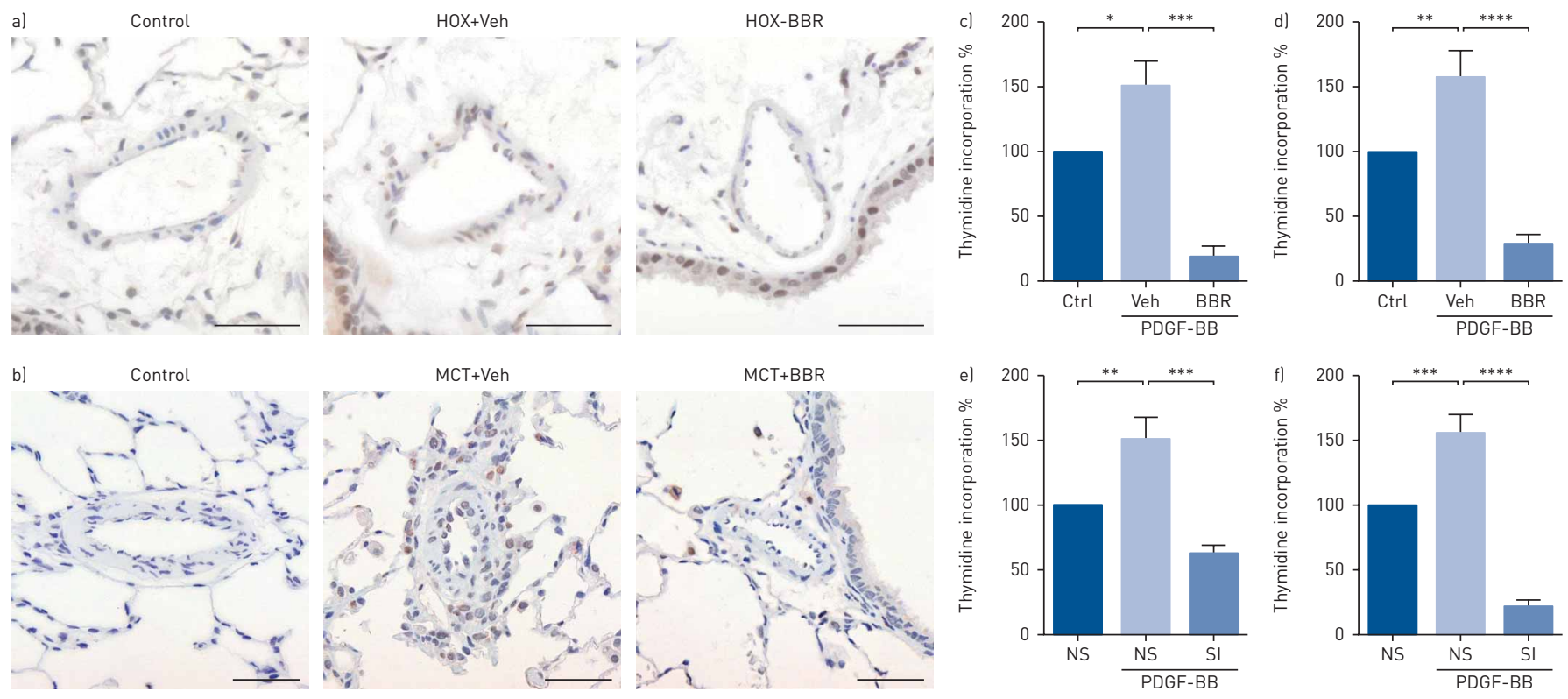
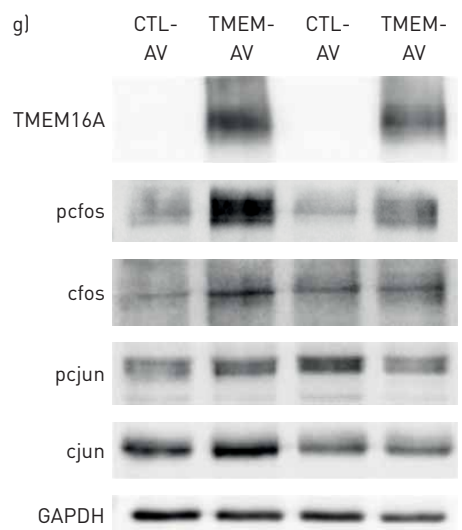

GAPDH
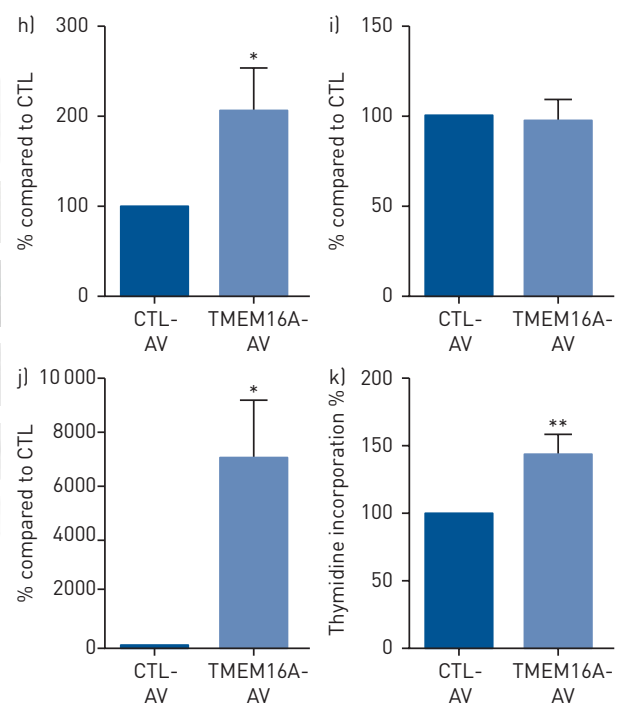

l)
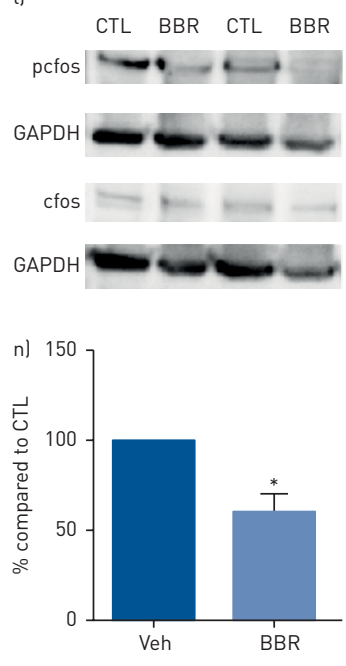

m)

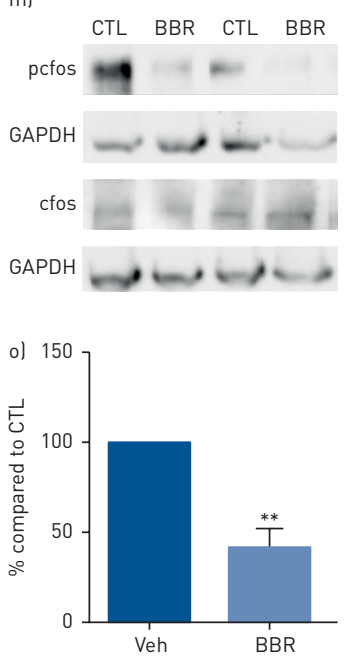

FIGURE 6 Role of TMEM16A in the proliferation of human pulmonary artery smooth muscle cells (PASMCs). a) Proliferating cell nuclear antigen (PCNA) staining (brown) of the pulmonary artery in mice that underwent 4 weeks of hypoxia and were treated either with vehicle (Veh) or benzbromarone (BBR) throughout weeks 3 and 4, compared to untreated normoxic controls. Scale bar $=50 \mu \mathrm{m}$. b) PCNA staining (brown) of the pulmonary artery of control and monocrotaline (MCT)-treated rats that received either Veh or BBR throughout weeks 3 and 4 after MCT treatment. Scale bar $=50 \mu \mathrm{m}$. c, d) Platelet-derived growth factor (PDGF)-BB induced proliferation of human donor (c) and idiopathic pulmonary arterial hypertension (IPAH) (d) PASMCs, measured with thymidine incorporation, in the absence (Veh) or presence of $30 \mu \mathrm{m}$ BBR (n=6 in all groups). Changes are expressed as percentage change compared to the untreated controls (Ctrl). e, f) PDGF-BB induced proliferation of human donor (e) and IPAH (f) PASMCs, measured with thymidine incorporation, $72 \mathrm{~h}$ after treatment with either non-silencing control RNA (NS) or TMEM16A siRNA (SI, $n=6$ in all groups). Changes are expressed as percentage change compared to the controls treated with non-silencing control RNA only (NS). *: $p<0.05 ;{ }^{* *}: p<0.01 ;{ }^{* * *}: p<0.001 ;{ }^{* * *}: p<0.0001$; one-way ANOVA with Bonferroni's multiple comparison test. g) Representative Western blots of PASMCs overexpressing CTL plasmid or TMEM16A plasmid via adenoviral application (AV), blotted for TMEM16A, phosphorylated cfos (pcfos), total cfos, phosphorylated cjun (pcjun), cjun and GAPDH. h-j) Summarised Western blot data of $n=7$ for pcfos/cfos (h), pcJUN/cJUN (i) and TMEM16A (j), compared to control (CTL). k) Overexpression of TMEM16A increased proliferation in PASMCs under basal conditions ( $n=8$ ), measured with thymidine incorporation. Changes are expressed as percentage change compared to the controls (CTL-AV). l, m) Representative Western blots of donor $(\mathrm{l})$ and IPAH $(\mathrm{m})$ PASMCs treated with or without BBR $(30 \mu \mathrm{M})$, blotted for TMEM16A, pcfos, total cfos and GAPDH. $\mathrm{n}, \mathrm{o})$ Summarised Western blot data of $n=7$ for pcfos/cfos for donor (n) and IPAH (o). *: $p<0.05$; **: $p<0.01$; unpaired t-test.

membrane of healthy donors' PASMCs to the range of IPAH patients. Because the intracellular $\mathrm{Cl}^{-}$ concentration in SMCs is relatively high [19], the corresponding reverse potential for $\mathrm{Cl}^{-}(-25 \mathrm{mV})$ is higher than the physiological resting membrane potential. Thus, opening of the TMEM16A channels results in $\mathrm{Cl}^{-}$efflux and subsequent membrane depolarisation, opening of the voltage-gated $\mathrm{Ca}^{2+}$ channels, $\mathrm{Ca}^{2+}$ influx and, consequently, PASMC contraction. BBR has the ability to inhibit URAT1 and xanthine oxidase, and is therefore a well-established medication for the treatment of gout [34]. It has 


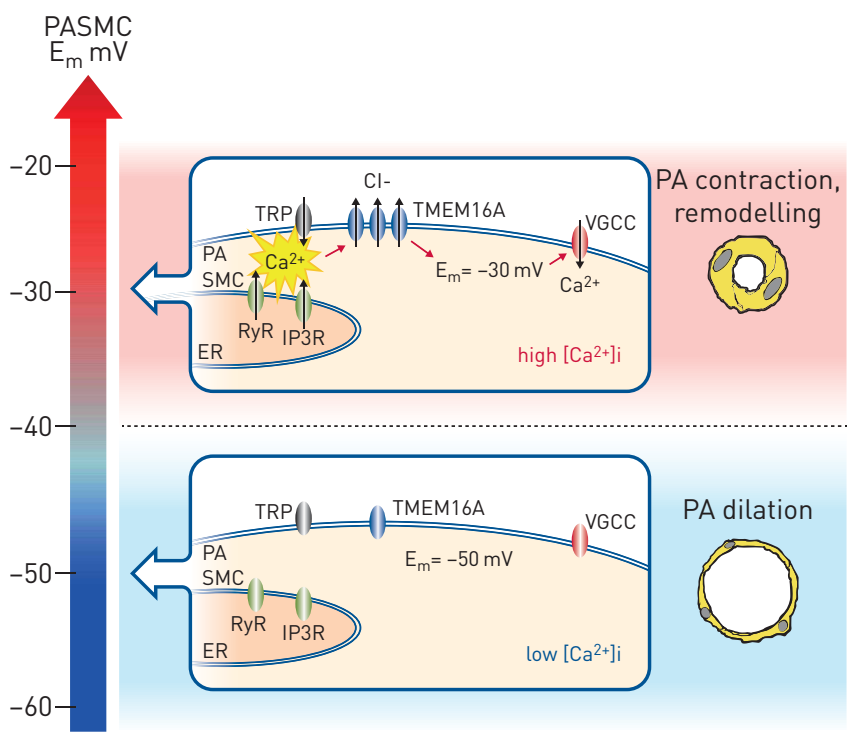

FIGURE 7 Effect of TMEM16A upregulation on the resting membrane potential in human pulmonary artery smooth muscle cells (PASMCs) and its pathophysiological consequences. The membrane potential (Em) of the PASMCs is the key to determining the intracellular $\mathrm{Ca}^{2+}$ concentration $\left[\mathrm{Ca}^{2+}\right]_{i}$ and the function of the pulmonary artery (PA). The Em of a healthy PASMC is around $-50 \mathrm{mV}$. Only a few TMEM16A channels are present and they are not activated. Owing to the negative $\mathrm{Em}$, voltage-gated $\mathrm{Ca}^{2+}$ channels (VGCC) are closed, and $\left[\mathrm{Ca}^{2+}\right]_{\mathrm{i}}$ is low. In contrast, the overexpression and increased activation of TMEM16A channels represent a depolarising current, raising $\mathrm{Em}$ to around $-30 \mathrm{mV}$. The subsequent VGCC opening increases $\left[\mathrm{Ca}^{2+}\right]_{\mathrm{j}}$, leading to PA contraction and PASMC proliferation. In addition, TMEM16A causes hyper-proliferation via cfos phosphorylation.

recently been identified as a potent TMEM16A blocker in a multi-drug screening for new TMEM16A inhibitors [18]. We chose BBR in our ex vivo and in vivo studies because this drug caused an IClCa inhibition similar to the siRNA treatment against TMEM16A, and its hyperpolarising effect was similar to the effect of TMEM16A silencing, demonstrating its potency as a TMEM16A channel blocker.

We show that BBR resulted in an ex vivo dose-dependent relaxation of pre-constricted mouse pulmonary artery, which is in line with its smooth muscle relaxing properties shown in the bronchi of asthmatic mice [18]. Moreover, we verified the acute haemodynamic effect of BBR in vivo in two chronic rodent models of PH. Our current clamp recordings provide evidence that BBR has no effect on the membrane potential of PASMCs isolated from healthy donor lungs. This suggests that in the healthy PASMCs, this channel is present at low levels with no significant effect on the resting membrane potential and the resting pulmonary artery tone. However, its pathologic upregulation and activation induces PASMC membrane depolarisation, pulmonary artery contraction and the resultant remodelling. Furthermore, TMEM16A might increase $\mathrm{Ca}^{2+}$ sensitivity of the PASMCs, given that a recent study has found a link between TMEM16A activity and the RhoA/ROCK kinases [35], and it is also known to amplify the store-operated $\mathrm{Ca}^{2+}$ entry in human PASMCs $[36,37]$.

Oral BBR is approved for preventive treatment of gout. In a pilot study in 10 IPAH patients undergoing right heart catheterisation, we applied a single maximal approved dose, but did not observe any acute vasodilatory effects. The lack of any acute effect was probably due to low BBR plasma concentrations and a very short exposure time. ET-1 receptor blockers, for example, although clinically highly effective, also have no acute haemodynamic effects. Chronic administration of BBR corresponding to therapeutic doses in models of hyperuricaemia in rodents or monkeys [24-26] improved the PH phenotype in two different rodent models. Our haemodynamic and echocardiographic data show that 4 weeks after $\mathrm{PH}$ induction, in the hypoxic mouse model and in the MCT rat model, BBR significantly attenuated the deleterious effects on right ventricular free wall thickness, cardiac index, pulmonary artery acceleration time, RVSP and Fulton index to be practically indiscernible from control levels. This suggests that BBR had strong beneficial effects on pulmonary vascular remodelling. The most likely molecular mechanism is the inhibition of TMEM16A by BBR. As a limitation of the study, we cannot exclude that the in vivo efficacy of BBR was in part mediated through effects on other pathways such as URAT1 and xanthine oxidase. However, we provide specific evidence for the TMEM16A pathway though the TMEM16A silencing and overexpression experiments in human PASMCs. Indeed, TMEM16A overexpression mimicked the features of IPAH-PASMCs, showing significantly increased PASMC proliferation via cfos phosphorylation. 
In conclusion, we found that the $\mathrm{Ca}^{2+}$-activated $\mathrm{Cl}^{-}$channel TMEM16A has a significant role in the pathologic mechanisms leading to chronic PASMC depolarisation, vasoconstriction and proliferation, which are all important features of IPAH. As a proof of principle, we demonstrated that TMEM16A inhibition by chronic BBR application strongly attenuates remodelling in two different rodent models of $\mathrm{PH}$, suggesting that TMEM16A is an important novel drug target for treatment of PAH.

Acknowledgements: We are grateful to Elvira Stacher and Zoltan Balint, who gave us valuable input on the design and execution of experiments, and Eugenia Lamont for proofreading the manuscript. The excellent technical help of Simone Tischler, Elisabeth Blanz, Sabine Halsegger, Lisa Oberreiter, Ida Niklasson and Verena Braunschmid is appreciated.

Author contributions: R. Papp, C. Nagaraj, D. Zabini, B.M. Nagy, M. Lengyel, D. Skofic Maurer, N. Sharma, P. Enyedi, G. Kovacs, H. Olschewski and A. Olschewski designed experiments, and acquired, analysed and interpreted data. B. Egemnazarov and M. Didiasova acquired and analysed data. G. Kwapiszewska, L.M. Marsh, A. Hrzenjak and P. Szucs contributed to the study design with ideas and/or technical advice. G. Höfler, M. Wygrecka, L.K. Sievers, B. Ghanim and W. Klepetko provided either infrastructure or experimental material. R. Papp, H. Olschewski, D. Zabini and A. Olschewski prepared the manuscript. C. Nagaraj, B.M. Nagy, G. Kovacs, G. Kwapiszewska, L.M. Marsh, A. Hrzenjak, M. Didiasova, M. Wygrecka and L.K. Sievers gave valuable advice on manuscript preparation.

Conflict of interest: C. Nagaraj has a patent (file number EP17169063.9): modulation of the calcium-activated chloride channel including TMEM16A represent a novel therapy for pulmonary hypertension, pending. D. Zabini has nothing to disclose. B.M. Nagy has a patent (file number EP17169063.9): modulation of the calcium-activated chloride channel including TMEM16A represent a novel therapy for pulmonary hypertension, pending. M. Lengyel has nothing to disclose. D. Skofic Maurer has nothing to disclose. N. Sharma has nothing to disclose. B. Egemnazarov has nothing to disclose. G. Kovacs reports personal fees and non-financial support from Actelion, Bayer, GSK, MSD, Boehringer Ingelheim, Novartis and Chiesi, and non-financial support from VitalAire, outside the submitted work. G. Kwapiszewska has nothing to disclose. L.M. Marsh has nothing to disclose. A. Hrzenjak has nothing to disclose. G. Höfler has nothing to disclose. M. Didiasova has nothing to disclose. M. Wygrecka has nothing to disclose. L.K. Sievers has nothing to disclose. P. Szucs has nothing to disclose. P. Enyedi has nothing to disclose. B. Ghanim has nothing to disclose. W. Klepetko has nothing to disclose. H. Olschewski reports grants, personal fees and non-financial support from Actelion, Bayer and Boehringer, personal fees and non-financial support from GSK, Chiesi, Menarini, TEVA, MSD and Ludwig Boltzmann Institute for Lung Vascular Research, personal fees from Novartis, AstraZeneca and Bellerophon, and grants and personal fees from Roche, outside the submitted work. A. Olshewski has a patent (file number EP17169063.9): modulation of the calcium-activated chloride channel including TMEM16A represent a novel therapy for pulmonary hypertension, pending. R. Papp has a patent (file number EP17169063.9): modulation of the calcium-activated chloride channel including TMEM16A represent a novel therapy for pulmonary hypertension, pending.

Support statement: A. Olschewski is supported by OeNB (16682), by the FWF (DK-MOLIN - W1241) and by the OeAD (TÉT 15-1-2016-0001). M. Lengyel was supported by the New National Excellence Program of the Ministry of Human Capacities (ÚNKP-18-3-III-SE-43). D. Skofic Maurer is supported by the FWF PhD Programme (DK-MOLIN W1241). N. Sharma is supported by the Molmed PhD programme of the Medical University of Graz. Funding information for this article has been deposited with the Crossref Funder Registry.

\section{References}

1 Tuder RM, Archer SL, Dorfmuller P, et al. Relevant issues in the pathology and pathobiology of pulmonary hypertension. Turk Kardiyol Dern Ars 2014; 42: Suppl. 1, 5-16.

2 Humbert M, Guignabert C, Bonnet $S$, et al. Pathology and pathobiology of pulmonary hypertension: state of the art and research perspectives. Eur Respir J 2019; 53: 1801887.

3 Kuhr FK, Smith KA, Song MY, et al. New mechanisms of pulmonary arterial hypertension: role of Ca2+ signaling. Am J Physiol Heart Circ Physiol 2012; 302: H1546-H1562.

4 Yuan JX, Aldinger AM, Juhaszova M, et al. Dysfunctional voltage-gated $\mathrm{K}+$ channels in pulmonary artery smooth muscle cells of patients with primary pulmonary hypertension. Circulation 1998; 98: 1400-1406.

5 Yuan XJ, Wang J, Juhaszova M, et al. Attenuated $\mathrm{K}+$ channel gene transcription in primary pulmonary hypertension. Lancet 1998; 351: 726-727.

6 Antigny F, Hautefort A, Meloche J, et al. Potassium channel subfamily k member 3 (KCNK3) contributes to the development of pulmonary arterial hypertension. Circulation 2016; 133: 1371-1385.

$7 \quad$ Ma L, Roman-Campos D, Austin ED, et al. A novel channelopathy in pulmonary arterial hypertension. $N$ Engl $J$ Med 2013; 369: 351-361.

8 Xia Y, Yang XR, Fu Z, et al. Classical transient receptor potential 1 and 6 contribute to hypoxic pulmonary hypertension through differential regulation of pulmonary vascular functions. Hypertension 2014; 63: 173-180.

9 Yu Y, Fantozzi I, Remillard CV, et al. Enhanced expression of transient receptor potential channels in idiopathic pulmonary arterial hypertension. Proc Natl Acad Sci USA 2004; 101: 13861-13866.

10 Zhang MF, Liu XR, Yang N, et al. TRPC6 mediates the enhancements of pulmonary arterial tone and intracellular $\mathrm{Ca} 2+$ concentration of pulmonary arterial smooth muscle cells in pulmonary hypertension rats. Sheng Li Xue Bao 2010; 62: 55-62.

11 Zhang S, Dong H, Rubin LJ, et al. Upregulation of $\mathrm{Na}+/ \mathrm{Ca} 2+$ exchanger contributes to the enhanced Ca2+ entry in pulmonary artery smooth muscle cells from patients with idiopathic pulmonary arterial hypertension. Am J Physiol Cell Physiol 2007; 292: C2297-C2305.

12 Song MY, Makino A, Yuan JX. STIM2 contributes to enhanced store-operated Ca entry in pulmonary artery smooth muscle cells from patients with idiopathic pulmonary arterial hypertension. Pulm Circ 2011; 1: 84-94.

13 Yuan XJ. Role of calcium-activated chloride current in regulating pulmonary vasomotor tone. Am J Physiol 1997; 272: L959-L968. 
14 Yang YD, Cho H, Koo JY, et al. TMEM16A confers receptor-activated calcium-dependent chloride conductance. Nature 2008; 455: 1210-1215.

15 Caputo A, Caci E, Ferrera L, et al. TMEM16A, a membrane protein associated with calcium-dependent chloride channel activity. Science 2008; 322: 590-594.

16 Schroeder BC, Cheng T, Jan YN, et al. Expression cloning of TMEM16A as a calcium-activated chloride channel subunit. Cell 2008; 134: 1019-1029.

17 Davis AJ, Shi J, Pritchard HA, et al. Potent vasorelaxant activity of the TMEM16A inhibitor T16A(inh) -A01. $\mathrm{Br} J$ Pharmacol 2013; 168: 773-784.

18 Huang F, Zhang $\mathrm{H}, \mathrm{Wu} \mathrm{M}$, et al. Calcium-activated chloride channel TMEM16A modulates mucin secretion and airway smooth muscle contraction. Proc Natl Acad Sci USA 2012; 109: 16354-16359.

19 Sun H, Paudel O, Sham JS. Altered expression of chloride transporters in rat pulmonary arterial smooth muscle associated with chronic hypoxic pulmonary hypertension. Circulation 2014; 130: Suppl. 2, A17752.

20 Manoury B, Tamuleviciute A, Tammaro P. TMEM16A/Anoctamin 1 protein mediates calcium-activated chloride currents in pulmonary arterial smooth muscle cells. J Physiol 2010; 588: 2305-2314.

21 Hoffmann J, Wilhelm J, Marsh LM, et al. Distinct differences in gene expression patterns in pulmonary arteries of patients with chronic obstructive pulmonary disease and idiopathic pulmonary fibrosis with pulmonary hypertension. Am J Respir Crit Care Med 2014; 190: 98-111.

22 Sala-Rabanal M, Yurtsever Z, Nichols CG, et al. Secreted CLCA1 modulates TMEM16A to activate $\mathrm{Ca}^{2+}$-dependent chloride currents in human cells. eLife 2015; 4: e05875.

23 Sala-Rabanal M, Yurtsever Z, Berry KN, et al. Modulation of TMEM16A channel activity by the von Willebrand factor type A (VWA) domain of the calcium-activated chloride channel regulator 1 (CLCA1). J Biol Chem 2017; 292: 9164-9174.

24 Ahn SO, Ohtomo S, Kiyokawa J, et al. Stronger uricosuric effects of the novel selective URAT1 inhibitor UR-1102 lowered plasma urate in tufted capuchin monkeys to a greater extent than benzbromarone. J Pharmacol Exp Ther 2016; 357: 157-166.

25 Cai HY, Wang T, Zhao JC, et al. Benzbromarone, an old uricosuric drug, inhibits human fatty acid binding protein 4 in vitro and lowers the blood glucose level in db/db mice. Acta Pharmacol Sin 2013; 34: 1397-1402.

26 Sun WF, Zhu MM, Li J, et al. Effects of Xie-Zhuo-Chu-Bi-Fang on miR-34a and URAT1 and their relationship in hyperuricemic mice. J Ethnopharmacol 2015; 161: 163-169.

27 Biasin V, Chwalek K, Wilhelm J, et al. Endothelin-1 driven proliferation of pulmonary arterial smooth muscle cells is c-fos dependent. Int J Biochem Cell Biol 2014; 54: 137-148.

28 Sun $\mathrm{H}$, Xia Y, Paudel O, et al. Chronic hypoxia-induced upregulation of $\mathrm{Ca}^{2+}$-activated $\mathrm{Cl}$ - channel in pulmonary arterial myocytes: a mechanism contributing to enhanced vasoreactivity. J Physiol 2012; 590: 3507-3521.

29 Forrest AS, Joyce TC, Huebner ML, et al. Increased TMEM16A-encoded calcium-activated chloride channel activity is associated with pulmonary hypertension. Am J Physiol Cell Physiol 2012; 303: C1229-C1243.

30 Wang K, Chen C, Ma J, et al. Contribution of calcium-activated chloride channel to elevated pulmonary artery pressure in pulmonary arterial hypertension induced by high pulmonary blood flow. Int J Clin Exp Pathol 2015; 8: $146-154$.

31 Hiram R, Rizcallah E, Sirois C, et al. Resolvin D1 reverses reactivity and Ca2+ sensitivity induced by ET1, TNF $\alpha$, and IL6 in the human pulmonary artery. Am J Physiol Heart Circ Physiol 2014; 307: H1547-H1558.

$32 \mathrm{Wu}$ MM, Lou J, Song BL, et al. Hypoxia augments the calcium-activated chloride current carried by anoctamin-1 in cardiac vascular endothelial cells of neonatal mice. Br J Pharmacol 2014; 171: 3680-3692.

33 Ferrera L, Caputo A, Ubby I, et al. Regulation of TMEM16A chloride channel properties by alternative splicing. J Biol Chem 2009; 284: 33360-33368.

34 Heel RC, Brogden RN, Speight TM, et al. Benzbromarone: a review of its pharmacological properties and therapeutic use in gout and hyperuricaemia. Drugs 1977; 14: 349-366.

35 Li RS, Wang Y, Chen HS, et al. TMEM16A contributes to angiotensin II-induced cerebral vasoconstriction via the RhoA/ROCK signaling pathway. Mol Med Rep 2016; 13: 3691-3699.

36 Yamamura A, Yamamura $\mathrm{H}$, Zeifman A, et al. Activity of $\mathrm{Ca}^{2+}$-activated $\mathrm{Cl}^{-}$channels contributes to regulating receptor- and store-operated $\mathrm{Ca}^{2+}$ entry in human pulmonary artery smooth muscle cells. Pulm Circ 2011; 1: 269-279.

37 Forrest AS, Angermann JE, Raghunathan R, et al. Intricate interaction between store-operated calcium entry and calcium-activated chloride channels in pulmonary artery smooth muscle cells. Adv Exp Med Biol 2010; 661: $31-55$. 